\title{
Présentation : Térence commenté, une école de rhétorique et de dramaturgie
}

Christiane Deloince-Louette et Jean-Yves Vialleton

\section{(2) OpenEdition}

1 Journals

Édition électronique

URL : http://journals.openedition.org/rhetorique/557

DOI : 10.4000/rhetorique.557

ISSN : 2270-6909

Éditeur

UGA Éditions/Université Grenoble Alpes

Édition imprimée

ISBN : 978-2-37747-029-7

\section{Référence électronique}

Christiane Deloince-Louette et Jean-Yves Vialleton, «Présentation : Térence commenté, une école de rhétorique et de dramaturgie », Exercices de rhétorique [En ligne], 10 | 2017, mis en ligne le 27 décembre 2017, consulté le 02 octobre 2020. URL : http://journals.openedition.org/rhetorique/557 ; DOI : https:// doi.org/10.4000/rhetorique.557

Ce document a été généré automatiquement le 2 octobre 2020.

\section{(c)}

Les contenus de la revue Exercices de rhétorique sont mis à disposition selon les termes de la Licence Creative Commons Attribution - Pas d'Utilisation Commerciale - Partage dans les Mêmes Conditions 4.0 International. 


\title{
Présentation : Térence commenté, une école de rhétorique et de dramaturgie
}

\author{
Christiane Deloince-Louette et Jean-Yves Vialleton
}

1 C'est dans la tragédie plutôt que dans la comédie qu'il nous semble aujourd'hui naturel d'aller chercher des «exercices de rhétorique ». De même, il semble que la théorie dramaturgique, à la suite de la Poétique d'Aristote (où manque, comme on le sait, la partie sur la comédie), se soit surtout consacrée à la tragédie, au point que l'on doute parfois qu'il existe une véritable théorisation du genre de la comédie ${ }^{1}$. Et pourtant, il faut en être bien conscient, c'est dans la comédie, plus précisément dans les comédies de Térence, et les comédies de Térence telles qu'elles étaient commentées par leurs maîtres d'école, que les auteurs de la Renaissance et de l'âge classique ont appris, enfants, et les bases de la rhétorique et celles de la dramaturgie. La critique, en particulier la critique américaine, a au demeurant depuis longtemps souligné cette importance des commentaires.

2 L'immense succès de Térence, et de Térence comme auteur scolaire, a tout d'abord été mis en évidence il y a presque un siècle par un spécialiste anglais de la littérature française, Harold Walter Lawton (1899-2005) dans sa thèse, Térence en France au XVI siècle. Contribution à l'histoire de l'humanisme en France (Paris, Jouve et $\mathrm{C}^{\mathrm{ie}}, 1926$; rééd. facsimilé Genève, Slatkine, $1970^{2}$ ), complété bien plus tard par un second tome sous-titré «Imitation et influence » (Genève, Slatkine, 1972³).

3 Le livre donne un répertoire bibliographique des éditions de Térence en Europe et des traductions de ses pièces en français «entre l'invention de l'imprimerie et 1600 ». Il dénombre plus de cent éditions des six comédies entre 1470 et 1500 , plus de 520 éditions au total avant 1600 (plusieurs travaux ultérieurs ont montré que ce nombre devait être même majoré, quasiment doublét) et une trentaine de traductions en français. Les premières éditions des comédies de Térence sont de 1470 (Strasbourg, non datée) et de 1471 (Venise). Les vers n'y sont pas distingués, ils le seront pour la première fois dans une édition de 1473. C'est aussi en 1473 que les actes sont pour la 
première fois distingués. Aux in-folio et in-quarto s'ajoutent très vite des in-octavo (1497) et même des éditions "de poche " (du in-12, 1528, au in-36), et des éditions séparées de pièces (le record est pour L'Andrienne, qui connaît quinze éditions entre 1470 et 1600$)$.

Dans les dernières lignes de la conclusion générale de l'ouvrage, Lawton donne comme un travail maintenant nécessaire l'évaluation de l'influence du succès énorme de cet auteur scolaire sur la littérature à partir de la Renaissance, en particulier sur le théâtre. Il annonce ainsi le second tome, dont les premiers chapitres portent sur l'étude de Térence à l'école, sur l'influence de Térence sur l'écriture dramatique, en particulier dans les théâtres néo-latin, italien et espagnol ; les chapitres suivants se concentrent sur la comédie en France au Xvi ${ }^{e}$ siècle, de l'Eugène de Jodelle à la fin du siècle.

5 Le travail programmé par Lawton a été fait pour le théâtre élisabéthain par un grand spécialiste américain de Shakespeare, Thomas Whitfield Baldwin (1890-1984) dans deux de ses ouvrages majeurs, William Shakspere's Smalle Latine and Lesse Greeke (Urbana, University of Illinois Press, 2 vol., 1944, rééd. 1966) et Shakspere's Five-Act Structure, Shakespeare's Early Plays on the Background of Renaissance Theories of Five-Act Structure from 1470 (Urbana, The University of Illinois Press, 1947, rééd. 1963).

6 Le premier livre reprend à son titre une formule célèbre de Ben Jonson, selon laquelle Shakespeare aurait connu bien peu de latin et encore moins de grec. Baldwin y fait un tableau des programmes scolaires dans l'Angleterre $\mathrm{du} \mathrm{xVI}^{\mathrm{e}}$ siècle et établit que Shakespeare a pu acquérir la culture humaniste et le savoir rhétorique dont témoignent ses pièces dans une simple "grammar school » à Stratford, ou une école équivalente, dans laquelle on n'étudiait certes pas de grec, mais beaucoup de latin (Ovide, les six premiers chants de l'Énéide, Tite-Live, Cicéron, Quintilien), et celui de Térence dès les premières classes («lower school»). Le livre consacre une section à Térence (vol. 1, III, B, XXVIII, p. 641 et suiv.), mais en parle très souvent ici et là (voir l'entrée « Terence » de l'index général, vol. 2, p. 769).

7 Dans le second livre, Balwin met en évidence la place primordiale donnée au comique latin dans l'éducation, en particulier en pays réformés. Térence est tellement étudié dans les classes de rhétorique qu'un manuscrit faisant la liste des professeurs d'un collège d'Oxford appelle les professeurs de philosophie, de logique et de rhétorique " Philosophie, logices et Terentij ${ }^{5} »$. Il rappelle aussi l'importance des commentaires à Térence, en particulier ceux des humanistes germaniques: quatre des trente-cinq chapitres du livre de Balwin (chap. VIII-XI) sont consacrés à ceux de Mélanchthon (Philipp Schwarzerd, auteur sous le nom de Philippus Mélanchthon), Barthélemy Masson (Latomus) et de Josse Willich (Jodocus Vuillichius), commentateurs particulièrement sensibles à la structure dramatique. Cet intérêt pour Térence en pays réformés est dû à Érasme. À la suite des humanistes italiens qui font de Térence le meilleur maître de conversation en latin, Érasme, grand admirateur de la pureté de la langue de Térence et de son urbanité, conseille à ses amis de l'avoir toujours sous la main (lettre 31), le prend en exemple pour expliquer sa méthode d'étude des classiques avec de jeunes élèves (De ratione studii et legendi interpretandique auctores, 1511) et en fait même un objet d'étude privilégié (épître dédicatoire de son édition des comédies de Térence aux deux fils du banquier polonais Seweryn Boner, Bâle, 1532). L'usage de Térence à l'école donne lieu cependant à des débats. Vivès souhaite le voir remplacé par des auteurs chrétiens (De disciplinis, 1531) et Térence ne sera pas mis en 1560 au programme de l'éducation jésuite (voir ici l'article de Sophie Conte). Reste qu'en 1764 
Diderot notera qu'un homme de lettres connaît son Térence "presque par cœur " (texte cité ici en ouverture de l'article d'Hélène Baby) : Térence n'a pas encore été tout à fait remplacé par Molière.

8 C'est dans ces commentaires scolaires que se façonne une théorie dramaturgique, dès avant même le retour et le triomphe d'Aristote, théorie dont héritent les théoriciens italiens de la dramaturgie, y compris, il faut le souligner, dans leur pensée de la tragédie (Giambattista Giraldi Cinthio, Lodovico Dolce).

9 Beaucoup des éditions de Térence sont en effet des éditions avec des introductions et un commentaire continu, selon une pratique d'édition des textes «classiques» qui a fait récemment l'objet de plusieurs études, dont un volume de 2013 publié aux Presses Universitaires de Louvain, contenant un article de Jan Bloemendal sur les Térence commentés ${ }^{6}$. Les manuscrits déjà offraient souvent des gloses ${ }^{7}$. Deux commentaires anciens avaient eu au Moyen Âge une grande fortune, l'un attribué à Servius, attribution contestée dès le $\mathrm{xVI}^{\mathrm{e}}$ siècle (analyse scène par scène imprimée la première fois en 1504 et jusqu'en 1544), l'autre œuvre d'Eugraphius (il contient des analyses rhétoriques et sera lui aussi imprimé), mais le commentaire qui devient la référence est celui de Donat (Ælius Donatus, grammaticus du IV siècle), connu en France en 1397, découvert au complet en 1433, publié pour la première fois en 1472. On attribue à Donat un traité sur le théatre, la comédie et la tragédie, presque toujours placé en introduction des éditions (en partie aujourd'hui attribué à Évanthius), une vie (en fait de Suétone), des arguments et un commentaire continu (aujourd'hui considéré comme une compilation hétérogène). Pour Le Bourreau de soi-même (Heautontimoroumenos), le commentaire de Donat, manquant, a été remplacé par un pastiche réalisé par Giovanni Calfurnio (Johannes Calphurnius Brixiensis), pour son édition de Donat publiée pour la première fois à Venise en 1472, et ensuite systématiquement repris. Mais on imprime aussi des commentaires modernes d'humanistes, c'est-à-dire la plupart du temps d'enseignants, ces commentaires prenant Donat comme point de départ. Avant 1600, plus de cinquante humanistes participèrent à l'établissement philologique du texte, la restitution et l'étude des vers, mais aussi à la rédaction de scholies constituant un commentaire continu, d'observations savantes ou d'" arguments", c'est-à-dire de simples résumés de l'histoire (comme ceux qu'on trouvait dans Donat) ou bien de véritables analyses de l'intrigue (comme ceux de Mélanchthon: voir ici l'article de Christiane Deloince-Louette). Les avant-propos signalent souvent que les efforts de ces éditeurs sont faits en faveur des jeunes gens (juvenes), ce qui indique que Térence fut "l'auteur par excellence des écoles et des collèges ${ }^{8}$ » et que ces éditions fournissaient sinon des éditions scolaires du moins des «livres du maître ». Les premiers commentaires modernes sont français : celui de Guy Jouenneaux (Guido Juvenalis) en 1492, réédité l'année suivante dans l'édition de Trechsel à Lyon (célèbre dans l'histoire du livre, notamment pour ses cent cinquante neuf belles gravures sur bois illustrant chaque scène ${ }^{9}$ ) avec l'ajout d' ' annotations » de Josse Bade (Ascensius), présentées dans l'avis au lecteur comme issues d'un enseignement donné à Valence et Lyon et explicitement destinées à la jeunesse et non aux savants (sur le commentaire de Bade, voir ici l'article de Laure Hermand-Schebat). Ces commentaires français connaissent une grande vogue avant d'être critiqués par les érudits postérieurs, puis abandonnés (après 1580). Suivent ceux de Paul Hemmerlin (Paulus Malleolus : Paris, annotations et arguments, 1499), réédités avec l'ajout des commentaires de Pietro Marso (Petrus Marsus : Strasbourg et Leipzig, 1503, édition neuf fois reprise jusqu'en 1519). En 1504 à 
Venise est publiée une édition, plusieurs fois reproduite, "avec cinq commentaires " (Terentius cum quinque commentis) : Donat, Josse Bade, Servius, Calfurnio, Jouenneaux (une gravure les représente comme des élèves au pied d'une chaire où professe un Térence couronné de lauriers); en 1517 paraît à Tübingen une autre édition où s'ajoutent notamment les notes de Hemmerlin. Sont publiés d'abord séparément en 1528 les commentaires et arguments de Mélanchthon et ceux de Christoph Hegendorf (Christophorus Hegendorphinius), en 1530 ceux d'Adriaan Van Baerland (Barlandus : sur ce commentaire, voir ici l'article de Mathieu Ferrand), en 1534 ceux de Latomus (dans une réédition de l'édition commentée par Mélanchthon), en 1540 les annotationes du Suisse Heinrich Loris (Glareanus), en 1540 les observationes de Dolet, en 1550 le commentaire de Willich, en 1555 les annotations de Muret $^{10}{ }^{10}$.

10 Des éditions que Lawton appelle «encyclopédiques» compilent de nombreux commentaires. Une édition à Venise en 1545 (de nombreuses fois rééditée ou copiée) réunit Donat, Calphurnius, Mélanchthon, Érasme, Goveanus, Barlandus, Marso, Rivius, Dolet, Glareanus, Hegendorf (pour des arguments), Latomus (arguments et notes). Les commentaires encadrent le texte et occupent même souvent la page entière ${ }^{11}$. À Lyon paraît en 1560 l'édition "triplex» (Terentius in quem triplex edita...) préparée par Pierre Daventès (Antesignanus), c'est-à-dire une édition déclinée en trois versions. Elle réunit quasiment tous les commentaires disponibles fondus en un même commentaire continu, donnant également en marge pour chaque vers son analyse métrique. Une page en début de chaque volume recense les dix-huit auteurs compilés, avec l'initiale qui permettra au lecteur de savoir de qui est la partie du commentaire qu'il lit. Le premier nom est celui de Mélanchthon, dont on reconnait le commentaire à l'absence d'initiale. Dans la première version (383 p.), le commentaire continu est dans les deux marges ; dans la seconde, il est plus développé ( 850 p.) et placé en plus en bas de page, en deux colonnes, sous le texte latin, occupant parfois presque entièrement la page. La troisième version ( $332 \mathrm{p}$.) est au contraire très allégée pour le commentaire, mais offre en bas de page sur deux colonnes une traduction continue en français, traduction littérale doublée par endroit d'une traduction littéraire. Dès 1548, une édition italienne (Il Terenzio latino commentato in lingua toscana..., Venise, 1548) proposait déjà des éclaircissements encadrant le texte et offrant une traduction littérale, syntagme par syntagme. Entre 1566 et 1568, Stephen Reich (Riccius) publie en trois tomes les préfaces (prolegomena) et commentaires accumulés par les titulaires de la chaire sur Térence instituée à Wittenberg par le duc de Saxe (Mélanchthon, Ambrosius Berndt, Johannes Strigelius...), avec des scholies en latin et allemand. C'est dans l'esprit de ces « éditions encyclopédiques » que nous proposons ici dans la rubrique "Atelier » une compilation de commentaires de la scène 3 de l'acte III de L'Andrienne et leur traduction, dans une présentation cependant différente des anciennes éditions. La présentation matérielle de ces livres, dont on peut aujourd'hui très facilement prendre connaissance grâce à la mise en ligne de leur numérisation en mode image, est cependant de grand intérêt, car, comme le remarquait Lawton, «l'aspect même d'un livre a sur le lecteur une influence psychologique qui n'est pas à négliger ${ }^{12}$ ».

11 Certains commentaires concernent la versification (Érasme, 1532, Loris, 1540), la philologie (Joannes Rivius, 1532), la lexicologie et l'érudition (Dolet, 1540), mais beaucoup sont des commentaires qui fournissent ce qu'on appellerait aujourd'hui une analyse littéraire, comme le faisait déjà Donat ${ }^{13}$. Certains (Mélanchthon, Willich) se focalisent particulièrement sur la rhétorique, mais aussi sur la morale (c'est-à-dire sur la psychologie humaine, utile à connaître pour l'orateur, mais plus utile encore pour 
former les jeunes âmes) et, comme le faisait déjà Donat, donnent des leçons de dramaturgie. L'épitre dédicatoire de Gregor Wagner en tête de l'édition commentée par Willich (1555, reprise dans le second volume de la Triplex), dont on trouvera le texte et la traduction dans la rubrique "Document", est significative de tout ce qu'un éducateur pouvait trouver chez Térence.

Les commentaires fondent à partir de Térence une véritable théorie de l'écriture dramatique. C'est ce qu'ont montré en détail plusieurs études parues peu après la publication des livres de Baldwin, dues en particulier à d'autres chercheurs américains de la même université.

Marvin Theodore Herrick (1899-1966), grand spécialiste de la dramaturgie à la Renaissance, en particulier en Italie, a fait la synthèse de ces leçons de dramaturgie et de rhétorique dans son Comic Theory in the Sixteenth Century (Urbana, The University of Illinois Press, Illinois studies in language and literature, vol. XXXIV, $\mathrm{n}^{\circ} 1$ et 2,1950 ).

Les leçons de rhétorique sont déjà largement présentes chez Donat (voir ici l'article de Bruno Bureau sur le commentaire de Donat à L'Hécyre) et concernent aussi l'actio (voir ici les articles de Sabine Chaouche et de Sophie Conte). Chez ses successeurs, elles portent sur l'élocution (Herrick donne un répertoire des figures de mot et de pensée présentes chez les commentateurs, p.189-214), mais aussi sur l'invention et la disposition. Herrick prend comme exemples (p. 179-188) les commentaires sur l'usage des preuves artificielles (signes, exemples, syllogismes, enthymèmes) ou indépendantes de l'art (témoignages, serments), et les commentaires où Willich applique à une scène entière, comme le dénouement de L'Eunuque, la division canonique du discours, de l'exorde à la péroraison (p. 6-8). Pour ce qui est de l'invention, on pourrait ajouter l'exemple des commentaires qui à la suite de Donat identifient dans les propos des personnages les « lieux » d'où ils tirent leurs arguments. Les commentateurs utilisent la grille des trois genres (délibératif, judiciaire, démonstratif), mais vont plus loin. Un simple choix d'un verbe de parole dans une glose ou dans un résumé de scène, d'apparence banale, sert parfois à indiquer précisément un jeu de langage particulier, jeu qui a sa procédure rhétorique ou qui en fixe le modèle et les variations possibles: Donat nous apprend par exemple que la demande (petitio ${ }^{14}$ ) utilise souvent un passage de délibération (Andria, v.315, Phormion, v. 485). Parfois le commentateur signale explicitement le caractère typiquement rhétorique du passage ( $u$ ut rhetoribus placet »). La rhétorique recoupe parfois l'art du dialogue, et donne en modèle des procédés qui seront mille fois utilisés, comme les répliques brèves pour rompre la monotonie d'une longue tirade (commentaire de Donat au vers 254 de L'Eunuque) ou l'acte débutant par une conversation déjà commencée (L'Eunuque, v. 391 ; Donat : " hic sermo sic prodit, ut post scaenam inchoatus esse noscatur ", " on entre dans cette conversation comme si elle avait commencé en coulisse »).

Les leçons de dramaturgie présentes dans le traité de la comédie de Donat et dans ses commentaires, puis dans les commentaires modernes comme ceux de Marso, Mélanchthon et Willich sont à la base de la théorisation du genre comique. Une large part de la matière de l'Explicatio eorum omnium quae ad Comoediae artificium pertinent (1548) de Francisco Robertello (dont Herrick donne en annexe la traduction, p. 227-239) vient de Donat. Ces commentaires, joints à la lecture de Cicéron et d'Horace, permettent une pensée de la fonction de la comédie et du comique. Les commentaires imposent aussi la conception ternaire de la structure dramatique, en exposition, nœud et dénouement, protasis, epitasis, catastrophe, en même temps que la nécessité de son 
unité d'ensemble, oeconomia, permise par l'usage savant des préparations de la suite et même du dénouement, paraskeuè (voir ici l'article de Christiane Deloince-Louette). La conception ternaire de la structure dramatique comique s'impose (même si Baldwin soutient que la répartition en cinq actes était présente chez Térence, contre l'idée qui prévaut actuellement, et concernait aussi la structure dramatique) jusqu'à être étendue à la pensée de la structure dramatique de la tragédie, en dépit d'Aristote : c'est ce que fait Giraldi dès le milieu du $\mathrm{xvI}^{\mathrm{e}}$ siècle (Discorsi, 1554). Les théoriciens essaient d'accorder Térence et Aristote (L'Hécyre modèle de la reconnaissance, de l'agnitio, selon par exemple Minturno dans son De poeta, 1559, et son Arte poetica, 1564). C'est encore sur Térence qu'on s'appuie en pleine époque classique lorsqu'il s'agit de réfléchir sur la règle aristotélicienne de l'unité de temps dans la tragédie et sur l'utilisation du temps nocturne (voir les débats sur Le Bourreau de soi-même qu'évoque ici l'article d'Hélène Baby).

16 C'est aussi de Térence commenté que provient la pensée moderne de l'intrigue, au sens étroit du terme, c'est-à-dire de la structure dramatique en tant que tissage de plusieurs histoires. Cinq des six pièces de Térence ont une structure dramatique double (fabula duplex, argumentum geminum), mettant en scène deux amoureux et aboutissant donc à un double dénouement, le second étant lié au premier : ainsi, le mariage de Pamphile avec Glycère, mais aussi de son ami Charinus, avec Philomène, dans L'Andrienne, modèle de comédie à « double fil » selon l'expression de l'abbé d'Aubignac dans sa Pratique du théâtre. Cette notion d'intrigue est propre à la comédie, mais elle va être généralisée. D'une part, elle va fonder la structure même de la tragi-comédie : Guarini se réfère au caractère « double » de L'Andrienne pour justifier l'intrigue de son Pastor fido (Compendio della poesia tragicomica, 1601). D'autre part, elle va s'étendre à la tragédie (Giraldi), alors que c'est une notion tout à fait étrangère à la Poétique d'Aristote qui impose au même moment son autorité. Les grands chefs-d'œuvre du théâtre de la Renaissance et de l'âge classique profiteront de cette leçon, produisant un argument double selon le procédé même de Térence, celui de la " contamination » de deux sources : Le roi Lear (l'histoire du roi et de ses filles tirée d'une chronique prise à Philip Sidney, celle de Gloucester et de ses fils) ou La vie est un songe (l'histoire de Rosaura, motif italien de la pèlerine amoureuse ; l'histoire du roi et de son fils, variation sur l'oracle d'(Edipe) pourraient servir d'exemples. Le renouvellement de la tragédie en France dans les années 1630 se souviendra lui aussi de cette leçon puisée dans la comédie. Quand Rotrou écrit son Hercule mourant (publié en 1636), il « contamine » deux pièces de Sénèque et en donne une version moderne parce que jouant sur un dénouement double, héroïque (l'apothéose d'Hercule), mais aussi amoureux (le mariage des jeunes premiers). La tragédie classique française héritera de l'intrigue d'origine comique, même si elle aura soin de penser l'un des fils comme un «épisode» de l'autre, pour obtenir l'unité d'action aristotélicienne (mais cette unité était déjà acquise dans L'Andrienne où la possibilité du mariage de Charinus ne pouvait exister que si Philomèle n'était pas mariée à Pamphile).

17 Les commentaires, comme l'a dit T. M. Herrick, portent aussi des leçons importantes sur la construction des personnages. T. M. Herrick y consacre un chapitre entier. Le sujet est approfondi par une autre étude parue elle aussi dans les Illinois studies in language and literature, l'année suivante (vol. XXXV, $\mathrm{n}^{\circ} 4$ ), celle d'Edwin W. Robbins, Dramatic Characterization in Printed Commentaries on Terence 1473-1600, Urbana, The University of Illinois Press, 1951. E. W. Robbins fournit la traduction de nombreux extraits des commentaires humanistes. Térence montre comment utiliser les 
personnages. Parmi les personnages secondaires, il signale le rôle du confident permettant d'éviter un monologue dans la première scène (persona protatica, comme Sosie dans L'Andrienne) et celui du personnage introduit pour permettre le dénouement (persona ad catastrophen machina, comme Criton dans la même pièce). À propos des personnages principaux, il montre par exemple comment un personnage peut susciter l'intérêt en ce qu'il est pris dans un dilemme cornélien avant la lettre. Le Pamphile de L'Andrienne, tel que l'analyse Mélanchthon dans ses Prolegomena, est soumis au double péril de désobéir à son père ou de trahir sa maîtresse. Térence fournit des exemples de personnages inventés conformément à la loi générale du decorum de l'Art poétique d'Horace, selon les paramètres qui conditionnent les mœurs (Cicéron en donnait onze ; Josse Bade en donne cinq dans ses Praenotamenta édités en 1502 ; Érasme en énumère seize dans l'Ecclesiastes), mais qui peuvent être aussi des personnages singularisés, car la vraisemblance générale n'est pas la vraisemblance particulière comme le souligne Érasme (le Pamphile de L'Andrienne, quoique jeune homme, est modéré ${ }^{15}$ ). Il donne le modèle de la création par combinaison paradoxale (ainsi Thaïs, la courtisane respectable, selon le commentaire de Donat au vers 198 de L'Eunuque), même si le procédé a été déjà utilisé avant lui par Plaute (ainsi Lydus, l'esclave sermonneur des Bacchides). Il donne aussi le modèle des effets de symétrie inversée entre personnages à la fois analogues et opposés (les deux vieillards et les deux jeunes gens de L'Andrienne, le doux Chrémès et le dur Simon, le sage et discret Pamphile et le Charinus puéril et imprudent). Les commentaires soulignent aussi ce que nous appellerions une approche fine de la peinture psychologique du personnage, qui dépasse le procédé littéraire, puisque, au-delà du divertissement, l'intérêt suprême de la comédie (et plus profondément ce qui justifie moralement qu'on incite les élèves à s'y intéresser, malgré les turpitudes qu'elle met sur scène) est de donner une image de la vie de tous les jours. De cette image on peut tirer un savoir (theseis, ethica, judicium, doctrina ${ }^{16}$ ) et ainsi apprendre à vivre tant qu'il n'est pas trop tard ${ }^{17}$. Au delà, la comédie a aussi un secret, celui de nous faire trouver dans les petites choses plus d'intérêt que dans les grandeurs tragiques, car «nous sommes nés non pour les troubles et les querelles, mais pour la paix et l'agrément ${ }^{18} »$. On pense à la morale de Montaigne (grand admirateur de Térence), dont il faudrait mieux voir qu'elle est « comique », comme son style.

E. W. Robbins note dès sa préface qu'étudier l'influence de Térence se limite trop souvent à la recherche des sources de telle ou telle comédie et qu'on néglige son influence en profondeur ( the fondamental bases of influence »). De fait, cette influence concerne l'art de la dramaturgie et dépasse de bien loin le genre de la comédie. Quand on s'interroge sur ce que Racine dans Phèdre doit à l'Antiquité, on se livre au jeu de la comparaison entre sa pièce et celles d'Euripide et de Sénèque (jeu devenu tradition scolaire) pour montrer l'originalité de Racine ou au contraire pour prouver que le vrai classicisme n'est pas français, mais antique, comme l'a fait le romantisme allemand (A. W. Schlegel, 1807). L'ouverture de la pièce de Racine vaut surtout pour le naturel de l'exposition, l'art subtil de la préparation (annonce et leurres, «ne nuda appareret

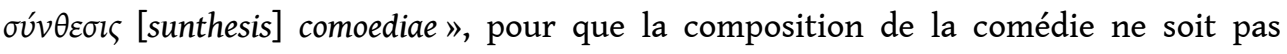
immédiatement visible, comme le dit Donat au vers 57 de Phormion) et la finesse psychologique (bienveillance du vieux gouverneur, pudeur adolescente et aspiration à la gloire du tout jeune homme), toutes choses qu'on peut apprendre chez Térence et non chez Sénèque. Le mythe de Phèdre étant connu par d'autres textes, Racine aurait pu écrire sa pièce sans qu'aient été transmises la pièce d'Euripide ni celle de Sénèque ; il ne l'aurait peut-être pas pu si l'on avait perdu les comédies de Térence. 


\section{BIBLIOGRAPHIE}

\section{Ouvrages et articles mentionnés}

BALDWIN, Thomas Whitfield, William Shakspere's Smalle Latine and Lesse Greeke, Urbana, University of Illinois Press, 2 vol., 1944, rééd. 1966, 753 p.

BALDWIN, Thomas Whitfield, Shakspere's Five-Act Structure, Shakespeare's Early Plays on the Background of Renaissance Theories of Five-Act Structure from 1470, Urbana, The University of Illinois Press, 1947, rééd. 1963, 848 p.

BLOEMENDAL, Jan, « In the Shadow of Donatus : Observations on Terence and Some of his Early Modern Commentators ", [in] ENENKEL, Karl et NELLEN, Henk (éd.), Neo-latin Commentaries and the Management of Knowledge in the Late Middle Age and the Early Modern Period (1400-1700), Louvain, Leuven University Press, 2013, p. 295-323.

BUCCHIONI, Umberto, Terenzio nel Rinascimento, Rocca S. Cascino, Cappelli, 1911.

GORDANI, Eleonora, « Térence », dans FURNo, Martine (dir.), La collection Ad usum Delphini, Grenoble, Ellug, 2005, vol. 3, p. 51-61.

GOYET, Francis, « Le problème de la typologie des discours », Exercices de rhétorique, 1 | 2013, en ligne (http://rhetorique.revues.org/122).

GUARDIA, Jean de, Poétique de Molière : comédie et répétition, Genève, Droz (« Histoire des Idées et Critique Littéraire ", vol. 431), 2007, 524 p.

HERMAND-SCHEBAT, Laure, « Texte et image dans les éditions latines commentées de Térence (Lyon, Trechsel, 1493 et Strasbourg, Grüninger, 1496) », Camenae (en ligne), nº 9, juin 2011, p. 1-14 (http://www.paris-sorbonne.fr/IMG/pdf/ARTICLE_9_Hermand-Schebat.pdf).

HERRICK, Marvin Theodore, Comic Theory in the Sixteenth Century, Urbana, The University of Illinois Press, Illinois studies in language and literature, vol. XXXIV, $\mathrm{n}^{\circ} 1$ et 2, 1950, $248 \mathrm{p}$.

HoLTz, Louis, « Le rôle des commentaires d'auteurs classiques dans l'émergence d'une mise en page associant texte et commentaire (Moyen-Âge occidental) », dans GOULET-CAZÉ, Marie-Odile et al. (dir.), Le commentaire entre tradition et innovation, Actes du colloque international de l'Institut des traditions textuelles (Paris et Villejuif, 22-25 septembre 1999), Paris, Vrin, 2000, p. 101-118. JAKOBI, Rainer, Die Kunst der Exegese in Terenzkommentar des Donat, Berlin/New York, Walter de Gruyter (Untersuchungen zur antiken Literatur und Geschichte, Bd. 47), 1996, rééd. 2013, 210 p.

LAWRENSON, Thomas Edward, « Les éditions illustrées de Térence dans l'histoire du théâtre, spectacle dans un fauteuil ?", dans JACQUOT, Jean (éd.), Le lieu théâtral à la Renaissance, Paris, CNRS, 1968, p. 1-23.

LAWTON, Harold Walter, Contribution à l'histoire de l'humanisme en France : Térence en France au XVI siècle, Paris, Jouve et Cie, 1926, 571 p. ; rééd. fac-similé Genève, Slatkine, 1970.

LAWTON, Harold Walter, Térence en France au XVI ${ }^{e}$ siècle. Contribution à l'histoire de l'humanisme en France, 2, Imitation et influence, Genève, Slatkine, 1972, 216 p.

MAROUZEAU, Jules, «Introduction » à TÉRENCE, Comédies, t. 1 [1 1 ère éd. 1942], Paris, Société d'édition «Les Belles lettres », Collection des universités de France, 1979, p. 17-22. 
ROBBINS, Edwin W., Dramatic Characterization in Printed Commentaries on Terence 1473-1600, Urbana, The University of Illinois Press (Illinois studies in language and literature, vol. XXXV, $n^{\circ} 4$ ), 1951, $162 \mathrm{p}$.

Roux, Maïté, Marc-Antoine Muret, lecteur et éditeur de Térence, mémoire de master 1, dir. R. Mouren, Université de Lyon 2, 2010, 312 p., http://www.ensib.fr/bibliotheque-numerique/documents/ 48932-marc-antoine-muret-lecteur-et-editeur-de-terence.pdf .

SABBADINI, Remigio, «Biografi e commentatori di Terenzio », Studi italiani di filologia, V, 1897, p. 281-327.

SCHLEGEL, August Wilhelm, Comparaison entre la Phèdre de Racine et celle d'Euripide, Paris, Tourneisen fils, 1807, 109 p. ; rééd. dans SCHLEGEL, August Wilhelm, Comparaison de la Phèdre de Racine et celle d'Euripide (et autres textes), édition présentée, annotée et commentée par Jean-Marie Valentin, Arras, Artois Presses Université, Artoithèque, 2013.

\section{NOTES}

1. Jean De Guardia, dans Poétique de Molière: comédie et répétition, Genève, Droz, 2007, relève que cette idée est répandue : "L'art de la comédie est conçu par une partie de la tradition critique (et surtout au $\mathrm{xx}^{\mathrm{e}}$ siècle, imprégné de poétique romantique), comme un art "purement de génie", sans règle et sans méthode » (p. 2) et affirme qu' " en réalité, les règles à propos de la comédie n'ont pas été écrites » (p. 3).

2. Avant lui, on pouvait consulter les bibliographies données dans des éditions de Térence, comme par exemple Londres, A. J. Valpy, 1824, vol. 3, p. 1694-1720 (encore utile pour les éditions après 1600) ainsi que diverses études comme R. Sabbadini, "Biografi e commentatori di Terenzio ", Studi italiani di filologia, V, 1897, p. 281-327, et U. Bucchioni, Terenzio nel Rinascimento, Rocca S. Cascino, Cappelli, 1911. - Pour les éditions du XVII ${ }^{\mathrm{e}}$ siècle, en particulier les éditions ad usum Delphini, voir le chapitre d'Eleonora Gordani, dans M. Furno dir., La collection Ad usum Delphini, Grenoble, Ellug, 2005, vol. 3, p. 51-61. L'édition Ad usum Delphini de Térence (par Nicolas Camus, Paris, Frédéric Léonard, 1675, copiée dix fois à Londres de 1688 à 1821) offre, non seulement une compilation des essais publiés sur Térence (d'Évanthius à Tanneguy Lefèvre), mais aussi une annotation nouvelle, destinée au débutants.

3. Sur Térence au collège, voir H. W. Lawton, Térence en France, op. cit. (dans le corps du texte), t. 2, p. 18 et suiv.

4. H. W. Lawton, Térence en France, ibid., p. 13.

5. T. W. Baldwin, Shakspere's Five-Act Structure, op. cit. (dans le corps du texte), note 2, p. 206.

6. J. Bloemendal, «In the Shadow of Donatus : Observations on Terence and Some of his Early Modern Commentators", dans K. Enenkel et H. Nellen éd., Neo-latin Commentaries and the Management of Knowledge in the Late Middle Age and the Early Modern Period (1400-1700), Louvain, Leuven University Press, 2013, p. 295-323.

7. Sur la «tradition térentienne ", voir J. Marouzeau, introduction à son édition de Térence, 1979 [1941], Paris, Les Belles Lettres (C. U. F.), t. 1, p. 17-22.

8. H. W. Lawton, Térence en France, op. cit., t. 1, p. 321.

9. Sur les illustrations de Térence, voir T. E. Lawrenson, « Les éditions illustrées de Térence dans l'histoire du théâtre, spectacle dans un fauteuil?", dans J. Jacquot éd., Le lieu théâtral à la Renaissance, Paris, CNRS, 1968, p 1-23 ; et L. Hermand-Schebat, « Texte et image dans les éditions latines commentées de Térence (Lyon, Trechsel, 1493 et Strasbourg, Grüninger, 1496) », Camenae (en ligne), $\mathrm{n}^{\circ} 10$, juin 2011, p. 1-12. 
10. Liste complète dans H.W. Lawton, Térence en France, op. cit., t. 1, p. 252-262 ; étude sur les commentaires, ibid., p. 291-319. Sur Muret et Térence, voir M. Roux, Marc-Antoine Muret, lecteur et éditeur de Térence, mémoire de master 1, dir. R. Mouren, Université de Lyon 2, 2010, http:// www.ensib.fr/bibliotheque-numerique/documents/48932-marc-antoine-muret-lecteur-et-

editeur-de-terence.pdf .

11. Cet encadrement du texte existait déjà dans les manuscrits : voir L. Holtz, " Le rôle des commentaires d'auteurs classiques dans l'émergence d'une mise en page associant texte et commentaire (Moyen-Âge occidental) ", dans M.-O. Goulet-Cazé et al. dir., Le commentaire entre tradition et innovation, Actes du colloque international de l'Institut des traditions textuelles (Paris et Villejuif, 22-25 septembre 1999), Paris, Vrin, 2000, p. 101-118.

12. H. W. Lawton, Térence en France, op. cit., t. 1, p. 283.

13. Sur les différents domaines sur lesquels portent les commentaires de Donat, voir les neuf chapitres de Rainer Jakobi, Die Kunst der Exegese in Terenzkommentar des Donat, Berlin, De Gruyter, 1996, rééd. 2013.

14. Sur la typologie des discours dans les traités de rhétorique modernes, fondée autant et plus sur les commentateurs antiques que sur les anciens traités de rhétorique, voir Fr. Goyet, « Le problème de la typologie des discours ", Exercices de rhétorique, 1|2013, en ligne (http:// rhetorique.revues.org/122).

15. Sur l'individualisation des personnages, voir E. W. Robbins, Dramatic Characterization..., op. cit. (dans le corps du texte), chap. 5, p. 91-95.

16. Les deux premiers mots sont utilisés dans son commentaire par Willich (1550), les mots latins par Vincentius Cordatus (1570).

17. Sur l'utilitas de la comédie, voir E.W. Robbins, Dramatic Characterization..., op. cit., chap. 5, p. 22-24.

18. Marso, commentaire à la fin de L'Andrienne, cité par E. W. Robbins, Dramatic Characterization..., ibid., p. 19.

\section{AUTEURS}

\section{CHRISTIANE DELOINCE-LOUETTE}

Université Grenoble Alpes - UMR Litt\&Arts / RARE Rhétorique de l'Antiquité à la Révolution

\section{JEAN-YVES VIALLETON}

Université Grenoble Alpes - UMR Litt\&Arts / RARE Rhétorique de l'Antiquité à la Révolution 